Somnologie $2018 \cdot 22: 76$

https://doi.org/10.1007/s11818-018-0150-z

Online publiziert: 17. Januar 2018

(c) Springer Medizin Verlag GmbH, ein Teil von

Springer Nature 2018

\title{
Danke an alle Gutachter 2017
}

Die Redaktion dankt allen Experten und Expertinnen für Ihre Unterstützung im Jahr 2017 als wissenschaftliche Gutachter für die Somnologie. Durch ihren Einsatz garantieren sie im Rahmen des Peer-Review-Verfahrens der eingereichten Manuskripte die Qualität der Zeitschrift.

Holger Hein

Clemens Heiser

Elisabeth Hertenstein

Jens Kerl

Sebastian Kerzel

Michael Klum

Nicolas Koranyi

Sylvia Kotterba

Ursula Kraemer

Dagmar Krefting

Geert Mayer

Georg Nilius

Meir Nitzan

Doris Oberle

Thomas Penzel

Roland Popp

Kurt Rasche

Kneginja Richter
Dieter Riemann

Felix Rosenow

Bernd Sanner

Melanie Schaedlich

Angelika Schlarb

Richard Schulz

Andreas Schulze-Bonhage

Kai Spiegelhalder

Jens Spiesshoefer

Boris A. Stuck

Tadas Stumbrys

Gerhard Weinreich

Hans-Günter Weeß

Niels Wessel

Thomas C. Wetter

Sandra Zimmermann

Peter Zimmermann

Robert Göder 